\title{
DEEP-UV BASED ACOUSTO-OPTIC TUNABLE FILTER FOR SPECTRAL SENSING APPLICATIONS
}

\author{
Narasimha S. Prasad \\ Nasa Langley Research Center, 5 N. Dryden St., MS 468, Hampton, VA 23681 \\ n.s.prasad@larc.nasa.gov
}

\begin{abstract}
In this paper, recent progress made in the development of quartz and KDP crystal based acousto-optic tunable filters (AOTF) are presented. These AOTFs are developed for operation over deep-UV to near-UV wavelengths of $190 \mathrm{~nm}$ to $400 \mathrm{~nm}$. Preliminary output performance measurements of quartz AOTF and design specifications of KDP AOTF are presented. At $355 \mathrm{~nm}$, the quartz AOTF device offered $~ 15 \%$ diffraction efficiency with a passband full-width-halfmaximum (FWHM) of less than $0.0625 \mathrm{~nm}$. Further characterization of quartz AOTF devices at deep-UV wavelengths is progressing. The hermetic packaging of KDP AOTF is nearing completion. The solid-state optical sources being used for excitation include nonlinear optics based high-energy tunable UV transmitters that operate around $320 \mathrm{~nm}$ and $308 \mathrm{~nm}$ wavelengths, and a tunable deep-UV laser operating over $193 \mathrm{~nm}$ to $210 \mathrm{~nm}$. These AOTF devices have been developed as turn-key devices for primarily for space-based chemical and biological sensing applications using laser induced Fluorescence and resonance Raman techniques.
\end{abstract}

Keywords: Deep-UV wavelengths; AOTF; chem-bio sensing

\section{Introduction}

NASA began Laser Risk Reduction Program (LRRP) in 2002 to develop reliable, robust, and compact laser technologies for lidar applications from space based platforms. The goal is to advance 1 micron and 2 micron lasers and associated wavelength conversion technologies for reliable operation in space environments. LRRP is a joint operation of Langley Research Center and Goddard Space Flight Center. The LRRP effort focuses on four Lidar techniques namely altimetry, Doppler, Differential Absorption Lidar (DIAL), and backscatter lidar. The emphasis is on six priority Earth Science measurements: (i) surface and ice mapping, (ii) horizontal vector wind profiling, (iii) carbon dioxide $\left(\mathrm{CO}_{2}\right)$ profiling, (iv) ozone $\left(\mathrm{O}_{3}\right)$ profiling, (v) aerosol/clouds, and (vi) river current monitoring.

To explore ozone profiling using DIAL technique from space based platforms, an highly efficient, allsolid-state, diode pumped, conductively cooled, single longitudinal mode and high-energy 1-micron to UV wavelength conversion technology to primarily generate UV wavelengths of $308 \mathrm{~nm}$ and $320 \mathrm{~nm}$ has been developed. This effort is a collaborative effort among Sandia National Labs, Fibertek, Inc. and NASA Langley Research Center ${ }^{1}$. This tunable UV transmitter technology has chem-bio sensing applications for NASA, defense, and Homeland Security Agency (HSA) missions. In the case of NASA, this UV converter technology provides a path for the development of compact, electrically controllable, and rugged chem-bio sensors specifically designed for investigating geological and mineralogical compositions as well as exploring precursors of life signature on various bodies of our Solar system. Besides NASA applications, this technology is suited for several HSA applications. For e.g., Tryptophan (amino acid generated by protein digestion), absorbs at $280 \mathrm{~nm}$ and emits over a range of 300-400 $\mathrm{nm}$ wavelengths with a resonance peak at $330 \mathrm{~nm}$.

Besides ozone related wavelengths, the LRRP UV transmitter technology is capable of generating tunable wavelengths ranging from $<200 \mathrm{~nm}$ to $400 \mathrm{~nm}$ spanning over deep-UV to near-UV spectral region using 
nonlinear optical techniques such as three-wave mixing. Among these wavelengths, deep-UV wavelength band is considered to play an important role in characterizing chem-bio species for space exploration missions. The tunable deep-UV wavelengths can serve as excitation wavelengths for Laser Induced Fluorescence (LIF) and Raman schemes including resonant Raman, and surface enhanced Raman effects. On the basis of this technology, a tunable deep-UV based chem-bio sensor technology has been being conceived primarily for space exploration purposes. Both, imaging and direct detection schemes corresponding to Laser Induced Fluorescence (LIF) and Raman techniques are being explored.

Various elements required for developing a robust, high resolution and electronically controllable chem-bio sensor are being investigated. One of the critical elements of this proposed tunable chem-bio sensor scheme is a narrow band tunable filter required to analyze spectral signatures emanating from targets of interest. For our initial studies, the acousto-optic tunable filter (AOTF) for analyzing Raman emissions due to deep-UV excitations has been selected. Besides effectively blocking background fluorescence, use of a deep-UV AOTF will allow electronic tuning of the peak passband to a desired emission wavelength. In this paper, the development of AOTFs appropriate for deep-UV wavelength operation is discussed. In Section 2, a brief description of excitation at deep-UV wavelengths is discussed. In Section 3, a brief overview on the ongoing development of UV transmitters as excitation sources is presented. In Section 4, the proposed chem-bio scheme is illustrated. In Section 5, design and fabrication of quartz crystal based AOTF is presented. In Section 6, initial performance measurements of the quartz AOTF are discussed. Summary and Conclusions are given in Section 7.

\section{Excitation at Deep-UV wavelengths}

The excitation in the deep-UV results in more intense Raman scattering. Deep-UV laser excitation has the ability to avoid fluorescence background in the Raman spectra. Tunability of the UV source could allow exploitation of resonance Raman effect. Resonance Raman Effect enhances the intensity of Raman lines. Deep-UV LIF is exceptionally sensitive to the presence of large biological molecules, bacteria, and organics. Recent work using UV LIF technique has shown that under suitable conditions, it is possible to detect as few as 5 to 10 bacteria on a surface, and zepta molar $\left\{10^{-21}\right.$ mole $\}$ of poly-aromatic hydrocarbons (PAHs). The specificity of Raman spectral molecular finger-prints has made the technique of great value in a number of areas including biochemistry and biophysics, early detection of cancers, chem-bio agents and for identifying minerals on planetary surfaces. LIF and laser Raman spectroscopy are considered sensitive techniques for providing reliable information on characteristics of organic molecules and the molecules of life. Use of excitation wavelengths below $250 \mathrm{~nm}$, endogenous and Raman emission of target molecules can be measured without mutual interference.

Abundant polycyclic aromatic hydrocarbons discovered in the fresh fracture surfaces of the Martian meteorite have stimulated new interest in the search for past life on Mars. These polyaromatic chains present in a Martian meteorite indicating probable past life signature. Deep-UV excitation of precursors such as kerogens and lignins could provide insight into biological precursors ${ }^{2}$. These are insoluble macromolecular hydrocarbons with low hydrogen content containing aromatic and polyaromatic components and are considered precursors for origins of life. Efforts are underway to devise sensitive techniques for the detection of lignins and kerogens.

\section{The UV Laser Transmitter}

The performance specifications of the single mode, conductively cooled, all-solid-state UV transmitter built under LRRP effort for ozone sensing applications include (i) nominal wavelength of $320 \mathrm{~nm}$ and 308 $\mathrm{nm}$, pulse energy of greater than $200 \mathrm{~mJ}$, pulsewidth around $25 \mathrm{~ns}$, and repetition frequency of $50 \mathrm{~Hz}$. For ozone sensing, the need for high pulse energy is to obtain enhanced performance during strong day light 
conditions. The basic scheme of this UV transmitter consists of a Nd:YAG laser operating at $1064 \mathrm{~nm}$ that pumps a nonlinear optics arrangement consisting of an Optical Parametric Oscillator (OPO) and a Sum Frequency Generator (SFG). The pump laser consists of a Nd:YAG gain medium based ring oscillator and four zig-zag Brewster angle slab amplifiers. Greater than $1 \mathrm{~J} /$ pulse at $1064 \mathrm{~nm}$ wavelength is obtained by amplifying the oscillator pulse energy of $\sim 30 \mathrm{~J}$ with four zig-zag slab amplifiers. Using the Second Harmonic Generation (SHG), the $532 \mathrm{~nm}$ wavelength is generated from $1064 \mathrm{~nm}$ wavelength. With KTP as a SHG crystal, up to $80 \%$ conversion efficiency has been demonstrated. The general scheme to generate UV wavelengths involves splitting the $532 \mathrm{~nm}$ wavelength energy almost equally using a beam splitter. One portion pumps a seeded KTP crystal based OPO to generate near-IR signal wavelength of $803 \mathrm{~nm}$. The other portion of $532 \mathrm{~nm}$ wavelength energy is mixed with $803 \mathrm{~nm}$ using a SFG crystal to generate 320 $\mathrm{nm}$ wavelength. In the case of $308 \mathrm{~nm}$, the OPO is designed to generate $731.5 \mathrm{~nm}$.

The OPO used for $532 \mathrm{~nm}$ pumped IR generation is known as Rotated Image Singly Resonant Twisted rectangle (RISTRA) type. The RISTRA OPO is a Four-mirror image-rotating non-planar ring resonator in a compact arrangement. The advantages of RISTA OPO include no alignment, long term stability, and good beam quality. The wall plug efficiency of the Nd:YAG pump laser is equal to $7 \%$ and the optical slope efficiency is equal to $11 \%$. The UV converter arrangement with $532 \mathrm{~nm}$ pump provides near-IR to UV wavelengths conversion efficiency of up to $24 \%$ conversion efficiency with extra cavity sum frequency generator arrangement. The semiconductor diode-pumped Nd:YAG pump laser with zigzag slab amplifiers allow robust and efficient design for use in space. The conduction cooled operation eliminates circulating liquids with in laser cavity. Finally, space-qualifiable component designs establish a path to space missions. The details of this setup are given in reference 1 .

The LRRP UV transmitter is a dual use technology that is suitable for many chem-bio applications. Besides ozone sensing, our objective is to develop an all solid-state deep (200-300 nm) UV laser transmitter and matched receiver unit that will be suitable for LIF and remote Raman techniques. The current tunable UV transmitter technology is anticipated to allow the generation of UV wavelengths ranging from $<200 \mathrm{~nm}$ to $400 \mathrm{~nm}$ with pulse energies of greater than $50 \mathrm{~mJ}$. The availability of large pump energy $>1 \mathrm{~J} /$ pulse at $1064 \mathrm{~nm}$ allows generation of sufficient energy at deep UV wavelengths for in-situ laser induced fluorescence experiments. The wavelength tunability of the UV source allows exploitation of resonance Raman effect that could enhance the intensity of Raman lines by 3 to 6 order of magnitude of aromatic amino acids, nucleic acids, and a variety of bacterial pigments. One of the methodologies to obtain wide range of UV wavelengths is by angle tuning one or more OPO and SFG crystals with appropriate seed wavelengths. Other efficient design possibilities are being explored. For our current experimental purposes, a deep-UV laser providing wavelengths from $193 \mathrm{~nm}$ to $210 \mathrm{~nm}$ has been procured and characterized from Coherent, Inc. The specifications of this deep-UV laser include PRF of $5 \mathrm{kHz}$, pulsewidth of $\sim 20 \mathrm{~ns}$ and spatial mode of $\mathrm{TEM}_{00}$. Deep UV wavelengths are obtained by fourth harmonic generation technique from a Nd:YLF laser pumped Ti:Sapphire laser arrangement. The modified transmitter coupled with a tunable filter is anticipated to be suitable for the following remote sensing techniques.

\section{The Chem-Bio Sensing Scheme}

The experimental arrangement for analyzing responses from chem-bio species due to deep-UV excitation under development is illustrated in Figure 1. In this scheme, a tunable deep UV laser transmitter is used for excitation. An acousto-optic tunable filter (AOTF) is utilized for UV LIF and resonance Raman Spectroscopic techniques. The fluorescence and Raman signals emanated from target species is collected using a telescope and selectively routed into an imaging spectrograph or an AOTF. The spectrally selective filtering is succeeded by detection and data processing tools that are commercially available. The proposed experimental scheme will be utilized for investigating mineralogical samples and/or simulants pertaining to 
extraterrestrial environments and for nondestructive detection of biologically important organisms and molecules. A secondary objective is to examine potential experimental schemes for planetary sample acquisition and habitability assessment as well as for human protection.

The experimental set-up has the flexibility to be adapted for both in-situ (dotted lines) and short-tomedium range (up to few $\mathrm{km}$ ) remote sensing. Furthermore, the proposed scheme allows for direct detection as well as imaging. Direct detection followed by imaging experiments based on LIF and resonance Raman provides species distribution features. In this setup, the digital CCD camera system is PIXIS 400B from Princeton Instruments and the UV detector is from Hamamatsu Corporation. A custom designed Cassegrain telescope for operation over deep-UV to visible region has been fabricated. For looking at visible returns, a commercially available AOTF operating in the visible region such as that made of $\mathrm{TeO}_{2}$ will be utilized. Characterization of critical components of this arrangement is progressing. In the following section, the characterization of AOTF devices is discussed.

\section{The Deep-UV AOTF device: Fabrication and Testing}

Acousto-optic (AO) effect is the diffraction of light by sound generated phase grating via photo elastic effect (change of refractive index with stress). AO effect provides a convenient way of controlling the frequency, intensity and direction of an optical radiation ${ }^{3-7}$. The basic principle of an AOTF device is illustrated in Figure 2. The dependence of optical passband on RF drive frequency gives rise to spectral filtering. In the case of an AOTF, an electronic tunable phase grating is established in an acousto-optic crystal due to the propagating ultrasonic wave ${ }^{8-10}$. Tuning is obtained by varying the RF drive frequency. AOTF devices are compact, solid-state devices with no moving parts. They provide good resolution $(<0.1$

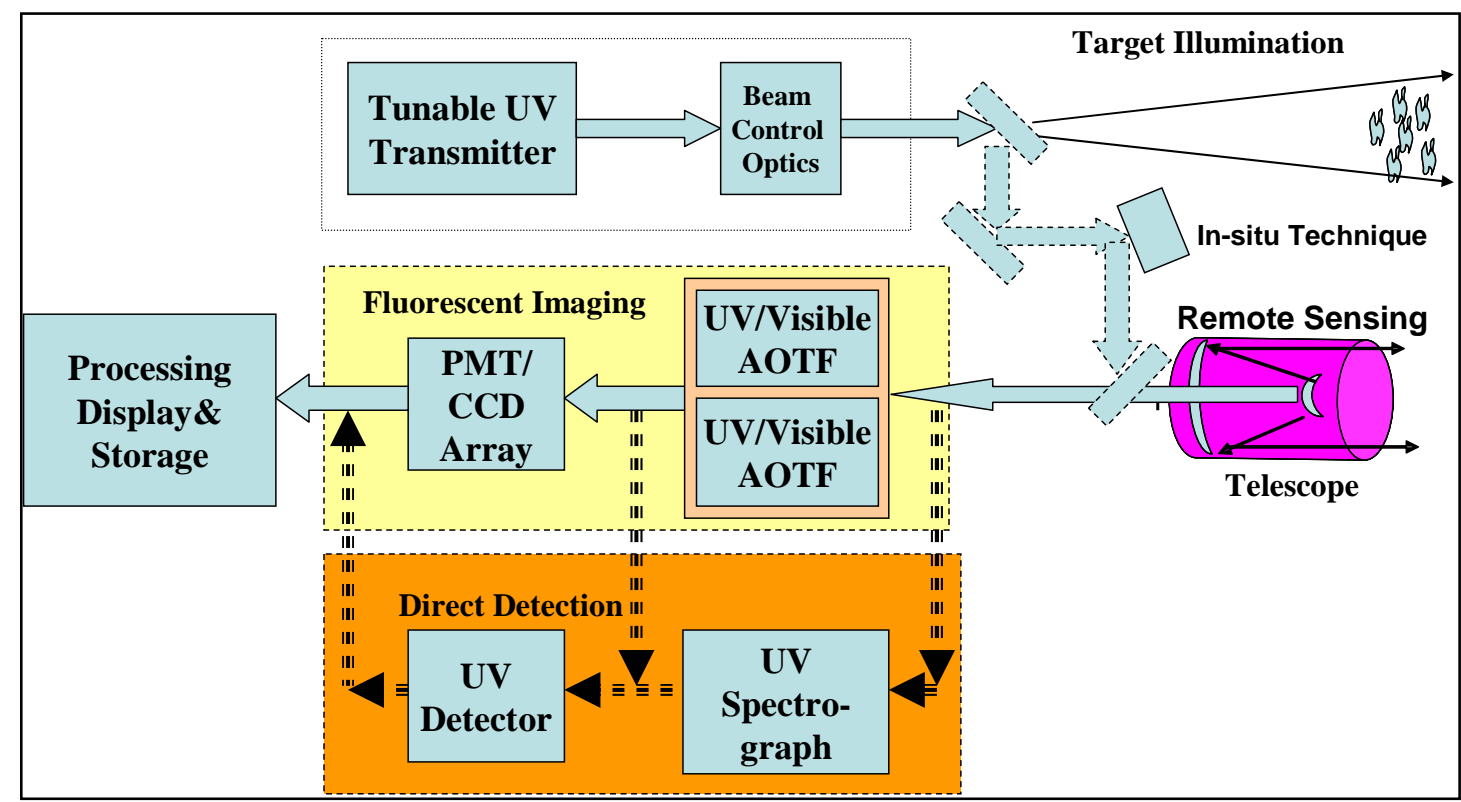

Fig. 1. Experimental setup for conducting chem.-bio sensing using single pixel detection as well as imaging schemes.

$\mathrm{nm}$ ) wavelength agility and rapid tunability via electronic means. Another aspect is programmability and random wavelength selection that allows remote operation. Other attractive features include low repeatability errors $(< \pm 0.05 \mathrm{~nm})$, high signal to noise ratio, reliability, and repeatability. AOTF devices allow spectral imaging over a wide spectral band. As such, AOTFs are becoming attractive for a wide range of applications including sensing and imaging applications in place of conventional gratings. If power consumption of few watts of RF power required to operate these devices is not an issue in a given 
operational environment, then AOTF devices could play a significant role. This is particularly true in the case of space missions.

An AO device can be operated either in Bragg or Raman-Nath regime. Bragg condition generates only one diffraction order. As such, Bragg cells are widely used in various applications. AOTFs can be constructed using collinear or non-collinear type of interactions in an anisotropic medium. Non collinear interactions allow operational flexibility as well as the diffracted order is well separated from the zero-order beams. Collinear interactions provide large apertures. However, one of the main disadvantages of collinear case is that the diffracted and zero-order beams are very close to each other and hence need a polarizing beam splitter to separate them. AOTFs generally use shear-mode interactions to optimize diffraction efficiency and passband characteristics.

AOTFs operating in the visible and Near-IR spectral regions have been well established. Practical deep-UV, mid-wave IR (3-5 microns) and long wave IR (8-12 microns) AOTFs are under development. Materials growth concerns are hampering their progress. However, steady progress is made in the case of long-wave IR materials for AOTF applications ${ }^{11}$. Recently, a near-UV AOTF operation using KDP crystal was reported ${ }^{12}$. In the following section, the design and measurements of a deep-UV AOTF is discussed.

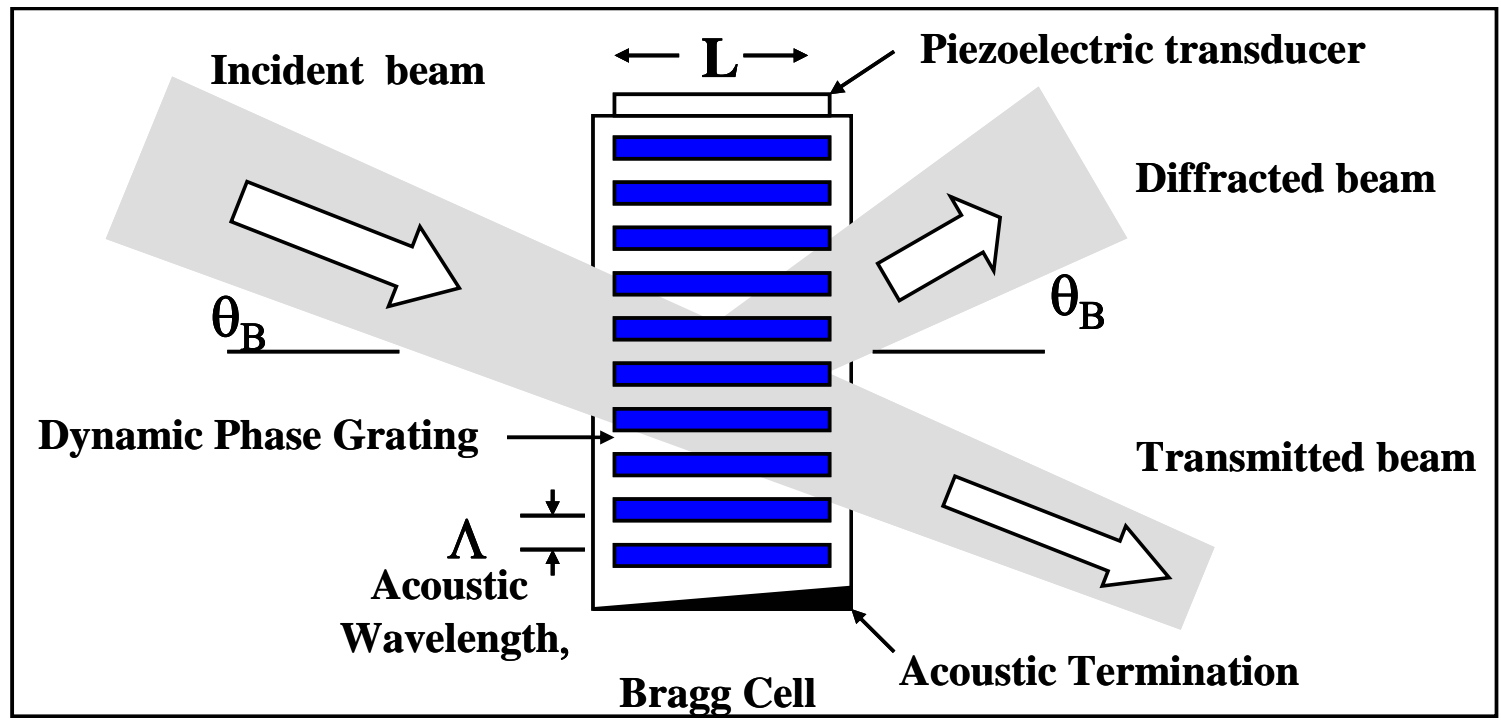

Fig. 2. The basic AOTF operational principle.

For deep-UV operation, design configuration using quartz and KDP materials were carefully studied. The AOTF units were fabricated by Brimrose Corporation. In this paper, the preliminary results of the quartz AOTF is presented while the KDP AOTF characteristics that are being hermetically packaged will be presented soon. Hermetical packaging is being done to prevent degradation. Both collinear and noncollinear device configurations were theoretically investigated. Generally, non-collinear configuration is preferred for AOTF operation due to relatively high diffraction efficiency and the advantage of having sufficiently separated output beams. In the case of non-collinear configuration design analysis, the angle 
between the zero-order and diffracted beam for quartz crystal was found to be around $0.5^{0}$. Since, no significant advantages with non-collinear design configuration was evident, collinear configuration was selected for quartz AOTF. The cut angle of the quartz AOTF crystal was $10^{\circ}$. Table 1 shows the test results along with design specifications. Although, the deep-UV laser was available for testing, no polarizers were available at the time of testing. Quartz AOTF characterization was carried out at $355 \mathrm{~nm}$ due to the availability of a quarter wave plate for separation of orthogonally polarized zero order and the diffracted beams. Hence, tripled Nd:YAG laser providing $355 \mathrm{~nm}$ was utilized for our initial characterization. The PRF of the Nd:YAG laser was $10 \mathrm{~Hz}$ and the pulsewidth was less than $10 \mathrm{~ns}$. A camera with spectral response ranging from $300 \mathrm{~nm}$ to 1 microns was utilized to capture output radiation from the AOTF device.

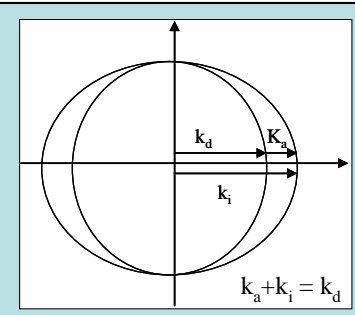

(a)

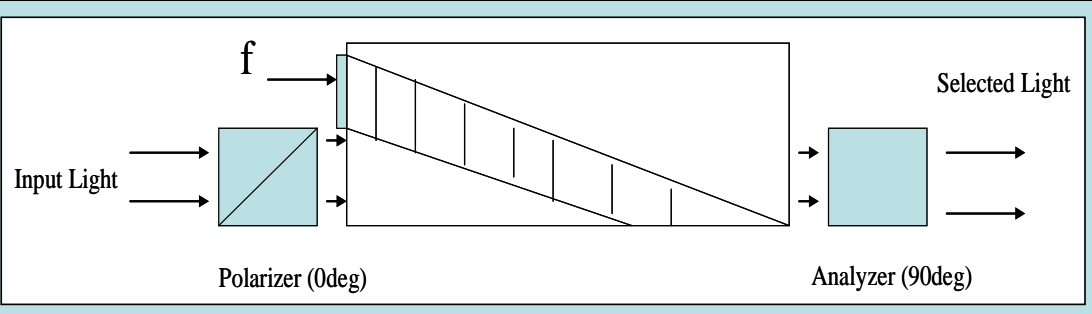

(b)

Fig. 3. Left: The collinear phasematching condition in relation to refractive indexe ellipsoids. Right: The collinear AOTF arrangement.

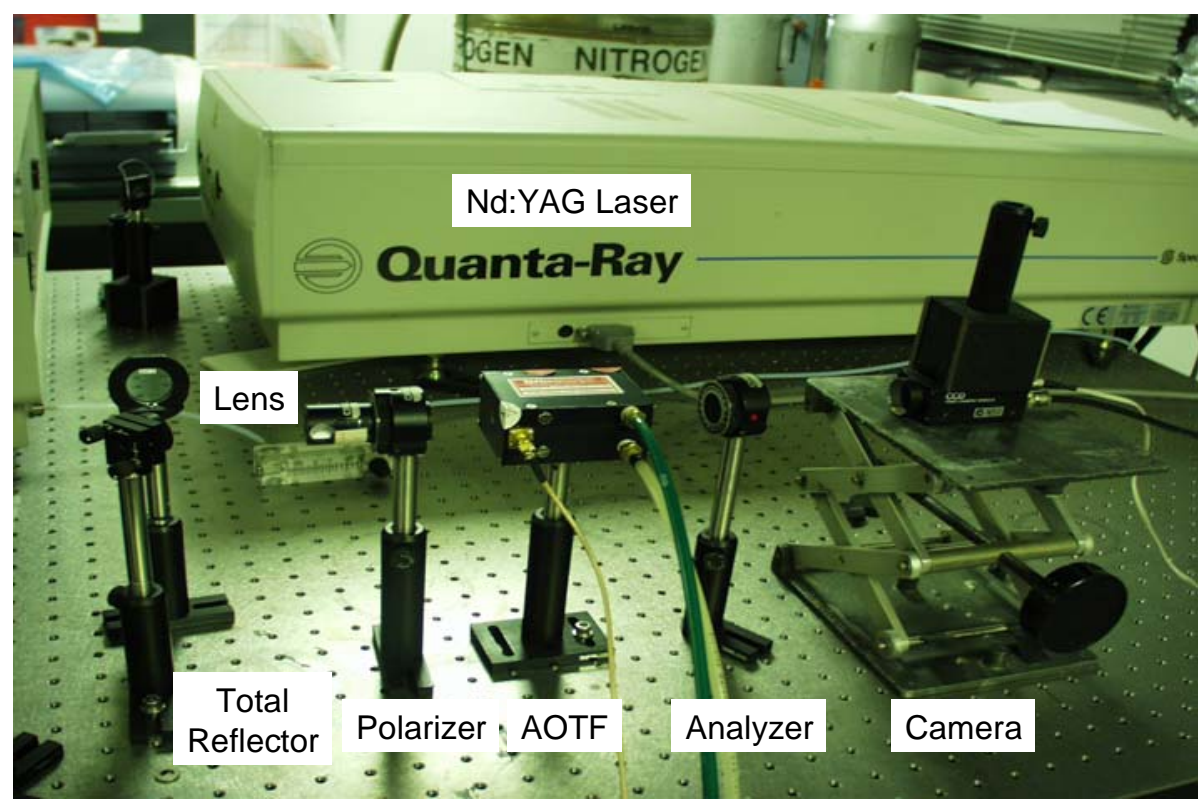

Figure 4. Experimental arrangement using a $355 \mathrm{~nm}$ wavelength obtained from a tripled Nd:YAG laser system. 


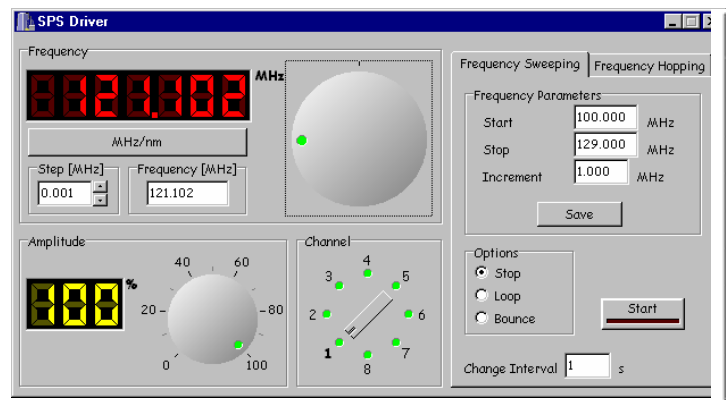

\begin{tabular}{|c|c|}
\hline Model & VFB-130-110-SPS-A-F15 \\
\hline Frequency Range & 75 to $185 \mathrm{MHz}$ \\
\hline Frequency Step Size & $15.625 \mathrm{~Hz}$ \\
\hline Frequency Accuracy & $0.010 \%$ absolute $(100 \mathrm{ppm}) ; 0$ to +60 deg. C \\
\hline Frequency Switching Speed & $15 \mathrm{~ms}$ typ (between $\mathrm{f}_{\text {min }}$ to $\left.\mathrm{f}_{\text {max }}\right)$ \\
\hline Phase Noise & $<-45 \mathrm{dBc} / \mathrm{Hz}$ @ $1 \mathrm{KHz}$ in $1 \mathrm{~Hz}$ bandwidth \\
\hline Power Output & 15 Watts max. \\
\hline Modulations & None (TTL or Analog Optional) \\
\hline Output Impedance & 50 ohms \\
\hline Output Connector & SMA jack on front panel \\
\hline
\end{tabular}

Figure 5. The AOTF control electronics box: Left: Graphical user interface. Right: Control system. specifications. (Courtesy of Brimrose Corporation).

Table 1. Design specifications of fabricated quartz and KDP AOTF devices.

\begin{tabular}{|l|c|c|c|}
\hline \multicolumn{1}{|c|}{ Parameter } & Units & S & Specifications \\
\hline Substrate & $\mathrm{NA}$ & Quartz & KDP \\
\hline Spectral Range & $\mathrm{nm}$ & $200-400$ & $190-450$ \\
\hline RF Frequency Range & $\mathrm{MHz}$ & $82-164$ & $75-185$ \\
\hline Optical Aperture & $\mathrm{mm}$ & $5.0 \times 5.0$ & $5.0 \times 5.0$ \\
\hline Acceptance Angle & Deg. & $5.4-7.6$ & $5.4-7.6$ \\
\hline Separation Angle & Deg. & -0.5 & $\sim 0.5$ \\
\hline Drive Power & Watts & 15 & 15 \\
\hline & & $0.4 @ 300 \mathrm{~nm}$ & \\
Spectral Resolution & $\mathrm{nm}$ & $0.8 @ 400 \mathrm{~nm}$ & $<0.1$ \\
\hline Didffraction Eff. At the peak & $\%$ & $15-20$ & $15-20$ \\
\hline Polarization & $\mathrm{NA}$ & Linear & Linear \\
\hline RF Connector & $\mathrm{NA}$ & SMA & SMA \\
\hline & & & Hermetically \\
Packaging & $\mathrm{NA}$ & Water Cooled & sealed \\
\hline \multicolumn{2}{|c|}{} & & \\
\hline
\end{tabular}

The AOTF was water-cooled for safety purposes. The first and zero order beams are almost collinear. Hence, a polarizer and an analyzer comprising of linear polarizers placed at right angles relative to each other, one before and one after the AOTF are required for beam separation. In the above table, spectral resolution is measured with collimated output. Diffraction efficiency refers to the laser line. The expected efficiency at the edges is around $10 \%$ of the peak value. Further characterization of this AOTF is planned with deep-UV wavelengths in coming days

\section{Experimental Results}

Measurements of AOTF performance were carried out at $355 \mathrm{~nm}$. The throughput of the quartz AOTF device was $>99 \%$. Up to 400 images per wavelength were collected, processed, and analyzed. From the image response, the passband characteristics were determined. In the best case, full width at half maximum (FWHM) less than $0.0625 \mathrm{~nm}$ was achieved. From the second set of measurements, a FWHM of $0.125 \mathrm{~nm}$ obtained. These passband characteristics are shown in Figure 4 along with the RF drive frequency in the top axis. The measured diffraction efficiency of the device was $~ 15 \%$. 

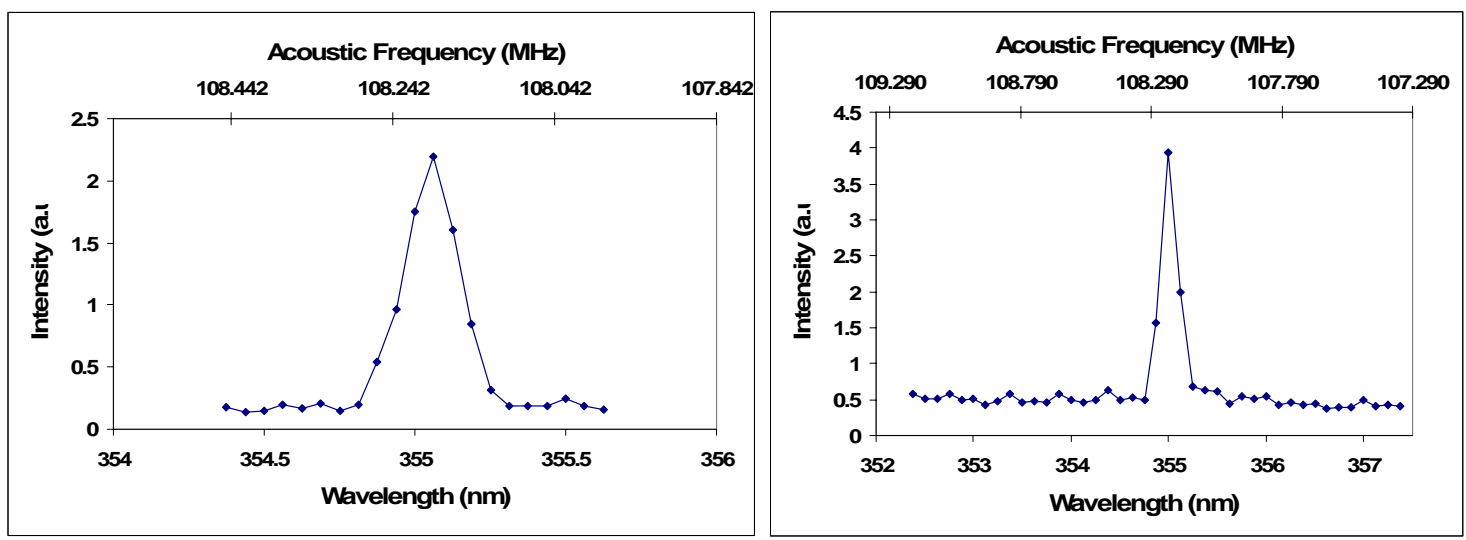

Fig. 6. Measured passband characteristics. Left Graph: Images per wavelength $=400$ and FWHM passband $=0.0625 \mathrm{~nm}$. Right Graph: Images per wavelength $=400$ and resolution $=0.125 \mathrm{~nm}$.

\section{Summary and Conclusions}

An all-solid-state Nd:YAG Pump laser has been developed with $>1 \mathrm{~J} /$ pulse at $50 \mathrm{~Hz}$ PRF and a pulsewidth of 22 ns. Using this pump laser, efficient high-pulse-energy, tunable UV wavelength generation technology has been demonstrated. This pump laser has the potential to generate deep-UV wavelengths. A chem-bio sensor scheme is being developed around this UV transmitter technology. Among several critical components, quartz and KDP based AOTFs have been designed and fabricated. Quartz AOTF has provided a passband of less than $0.0625 \mathrm{~nm}$ (FWHM) with 15\% diffraction efficiency. Currently, KDP AOTF is being hermetically packaged. In-situ and remote chem-bio sensing experiments are planned in the near future. Potential NASA applications include mineralogical investigations on the Moon, asteroids and planets.

\section{Acknowledgements}

The author would like to thank Dr. Jolanta Soos, Dr. Sudhir Trivedi and Dr. Feng Jin of Brimrose Corporation and Prof. Uwe Hommerich of Hampton University for useful discussions and support.

\section{9. $\quad$ Selected References}

1. Narasimha S. Prasad, Upendra N. Sing, Floyd E. Hovis, and Darrell J. Armstrong, "High energy, single-mode, allsolid state and tunable UV laser transmitter,” Proc. SPIE Vol. 6214, April 2006.

2. Narasimha S. Prasad, Richard E. Davis, Upendra N. Singh, "Sensing of kerogens and lignens using laser-induced fluorescence at deep-UV wavelengths,” Proc. SPIE Vol. 5887, Lidar Remote Sensing for Environmental Monitoring VI; Upendra N. Singh; Ed., Aug 2005.

3. R.M. Dixon, “Acoustic diffraction of light in anisotropic media, “ IEEE J. Quantum Electron., QE-3, 85-93 (1967).

4. S.E. Harris and R.W. Wallace, “Acousto-optic Tunable Filter,” J. Opt. Soc. Am., 59, 744-747 (1969).

5. A. Yariv and Yeh, “Optical Waves in Crystals,” Wiley, New York 1984.

6. J. Xu and R. Stroud, Acousto-Optic Devices, Jon-Wiley \& Sons, Inc., New York, 1992.

7. Neelam Gupta, “Acousto-optic Tunable Filters”, optics and Photonics News, November 1997, pp 23-27.

8. I.C. Chang, “Noncollinear acousto-optic filter with large angular aperture,” Appl. Phys. Lett. 25, 370-372 (1974).

9. I.C. Chang, "Collinear beam acousto-optic tunable filters," Electronics Lett. Vol 28, No 13 PP 1255-6 (1992). 
10. I.C. Chang, Tunable Acousto-Optic Filter Utilizing Acoustic Beam Walkoff in Crystal Quartz," AppI. Phys. Lett., 25, 323-4 (Sept. 1974).

11. Narasimha S. Prasad et. al, "Wide bandwidth long-wave IR acousto-optic filter for lidar applications," SPIE's Fourth International Asia-Pacific Environmental Remote Sensing Symposium, 8-11 November 2004, Honolulu, Hawaii, USA.

12. Vitaly Voloshinov and Neelam Gupta, “Ultraviolet-visible imaging acousto-optic tunable filters in KDP,” Applied Optics, Vol. 43, No. 19, 1 Jul 2004, pp. 3901-3909. 